\title{
Caracterização da performance do material Poliácido Lático (PLA) manufaturado pela tecnologia de Modelagem de Fusão e Deposição (FDM)
}

\author{
Performance characterization of Polylatic Acid (PLA) material manufactured by Fusion and \\ Deposition Modeling (FDM) technology \\ Caracterización del rendimiento del material de Poliácido Láctico (PLA) fabricado con tecnología \\ de Modelado de Fusión y Deposición (FDM)
}

Recebido: 18/06/2021 | Revisado: 29/06/2021 | Aceito: 04/07/2021 | Publicado: 15/07/2021

\author{
Rodolfo Ramos Castelo Branco \\ ORCID: https://orcid.org/0000-0002-9034-7985 \\ Universidade Federal de Campina Grande, Brasil \\ E-mail: rodolfo.ramos@nutes.uepb.edu.br \\ Ketinlly Yasmyne Nascimento Martins \\ ORCID: https://orcid.org/0000-0001-8653-8052 \\ Universidade Federal de Campina Grande, Brasil \\ E-mail: yasmyne.martins@nutes.uepb.edu.br \\ Anna Kellssya Leite Filgueira \\ ORCID: https://orcid.org/0000-0002-7739-3522 \\ Universidade Estadual da Paraíba, Brasil \\ E-mail: annakellssya21@gmail.com \\ Eduardo Jorge Oliveira Valadares \\ ORCID: https://orcid.org/0000-0003-4884-3093 \\ Universidade Estadual da Paraíba, Brasil \\ E-mail: edujvo@gmail.com \\ Kátia Elizabete Galdino \\ ORCID: https://orcid.org/0000-0002-2160-946X \\ Universidade Estadual da Paraíba, Brasil \\ E-mail: katiagaldino@gmail.com \\ Misael Elias de Morais \\ ORCID: https://orcid.org/0000-0003-3211-5104 \\ Universidade Estadual da Paraíba, Brasil \\ E-mail: moraes@gmail.com \\ Maria das Graças Ouriques Ramos \\ ORCID: https://orcid.org/0000-0002-3007-9851 \\ Universidade Estadual da Paraíba, Brasil \\ E-mail: ouriquesgr@gmail.com \\ Núbia do Nascimento Martins \\ ORCID: https://orcid.org/0000-0001-5941-3078 \\ Universidade Estadual da Paraíba, Brasil \\ E-mail: mate_matematicaa@gmail.com \\ Khelvyn Yhasley Nascimento Martins \\ ORCID: https://orcid.org/0000-0002-9954-1696 \\ Universidade Estadual da Paraíba, Brasil \\ E-mail: khelvyn.martins@hotmail.com \\ Jonh Kennedy Guedes Rodrigues \\ ORCID: https://orcid.org/0000-0003-0340-5567 \\ Universidade Federal de Campina Grande, Brasil \\ E-mail: profkennedy@hotmail.com
}

\begin{abstract}
Resumo
O objetivo deste estudo foi caracterizar a performance do material poli ácido lático (PLA) quando manufaturado pela tecnologia modelagem por fusão e deposição (FDM). Para a elaboração e organização do procedimento experimental, este estudo foi construído de acordo com a norma ASTM D3039/3039-95A, utilizando um total de 36 corpos de prova, modelados através do software CAD Autodesk Inventor e, posteriormente, exportados para o software CAM Makerware, onde definiu-se os parâmetros e processos para a impressão 3D. Os ensaios de tração foram realizados na máquina EMIC DL 3000, mantendo uma condição padrão com o laboratório a uma temperatura em torno de $20^{\circ} \mathrm{C}$. Os valores definidos como significativos para este estudo foram: Tensão Máxima; Tensão de Ruptura e Módulo de Elasticidade. Os corpos de prova manufaturados apresentaram comportamento anisotrópico. Nos testes de tensãodeformação, as rupturas ocorreram em perfeito rompimento de perfil. As curvas tensão-deformação apresentaram-se
\end{abstract}


praticamente sem área de escoamento. As taxas de deposição de $100 \%$ para o eixo X demonstraram maiores índices para tensão de ruptura em contraposto as taxas de deposição de $20 \%$ do eixo Y. No que se refere ao alongamento, as peças com $20 \%$ de taxa de deposição no eixo X apresentaram maiores índices. As propriedades mecânicas, resistência à tração e resistência à flexão, aumentaram conforme o preenchimento. O PLA, manufaturado pelo processo de FDM, demonstrou melhor performance mecânica quando produzido em eixo X com taxas de deposição de $100 \%$ para o preenchimento interno estrutural e temperatura de extrusão de $200^{\circ} \mathrm{C}$. Caracteriza-se como típico frágil polimérico.

Palavras-chave: Manufaturas; Impressão tridimensional; Processos mecânicos; Fenômenos mecânicos.

\begin{abstract}
The aim of this study was to characterize the performance of polylactic acid (PLA) material when manufactured by melt deposition modeling (FDM) technology. For the elaboration and organization of the experimental procedure, this study was constructed in accordance with the ASTM D3039/3039-95A standard, using a total of 36 specimens, modeled using Autodesk Inventor CAD software and later exported to CAM software Makerware, where parameters and processes for 3D printing were defined. The tensile tests were carried out on the EMIC DL 3000 machine, maintaining a standard condition with the laboratory at a temperature around $20^{\circ} \mathrm{C}$. The values defined as significant for this study were: Maximum Tension; Breaking Stress and Modulus of Elasticity. The manufactured specimens showed anisotropic behavior. In the stress-strain tests, the ruptures occurred in perfect profile rupture. The stressstrain curves showed practically no yield area. The deposition rates of $100 \%$ for the $\mathrm{X}$ axis showed higher rates for tensile strength as opposed to the $20 \%$ deposition rates for the Y axis. With regard to elongation, the parts with $20 \%$ deposition rate on the $\mathrm{X}$ axis had higher rates. Mechanical properties, tensile strength and flexural strength, increased with filling. PLA, manufactured using the FDM process, demonstrated better mechanical performance when produced on an $\mathrm{X}$ axis with $100 \%$ deposition rates for the structural internal fill and an extrusion temperature of $200^{\circ} \mathrm{C}$. It is characterized as a typical polymeric brittle.
\end{abstract}

Keywords: Manufactured materials; Printing, Three-dimensional; Mechanical processes; Mechanical phenomena.

\title{
Resumen
}

El objetivo de este estudio fue caracterizar el rendimiento del material de ácido poliláctico (PLA) cuando se fabrica mediante la tecnología de modelado de deposición por fusión (FDM). Para la preparación y organización del procedimiento experimental, este estudio se construyó de acuerdo con la norma ASTM D3039 / 3039-95A, utilizando un total de 36 probetas, modeladas con el software Autodesk Inventor CAD y posteriormente exportadas al software CAM Makerware, donde parámetros y Se definieron los procesos para la impresión 3D. Se realizaron ensayos de tracción en la máquina EMIC DL 3000, manteniendo una condición estándar con el laboratorio a una temperatura en torno a $\operatorname{los} 20^{\circ} \mathrm{C}$. Los valores definidos como significativos para este estudio fueron: Voltaje Máximo; Esfuerzo de rotura y módulo de elasticidad. Las probetas fabricadas mostraron un comportamiento anisotrópico. En las pruebas de tensión-deformación, las roturas se produjeron en perfecta rotura de perfil. Las curvas de tensión-deformación no mostraron prácticamente ningún área de fluencia. Las tasas de deposición del 100\% para el eje X mostraron tasas más altas de resistencia a la tracción en comparación con las tasas de deposición del $20 \%$ para el eje Y. Con respecto al alargamiento, las piezas con una tasa de deposición del $20 \%$ en el eje X tenían tasas más altas. Propiedades mecánicas, resistencia a la tracción y resistencia a la flexión, aumentadas con el relleno. El PLA, fabricado mediante el proceso FDM, demostró un mejor rendimiento mecánico cuando se produjo en un eje X con tasas de deposición del $100 \%$ para el relleno interno estructural y una temperatura de extrusión de $200^{\circ} \mathrm{C}$. Se caracteriza por ser un frágil polimérico típico.

Palabras clave: Materiales manufacturados; Impresión tridimensional; Procesos mecánicos; Fenómenos mecánicos.

\section{Introdução}

O Poli ácido lático (PLA), polímero biodegradável produzido a partir de recursos biológicos renováveis como amido e açúcares, devido seu perfil de propriedade e processabilidade termoplástica e biológica, tem-se demonstrado um elemento promissor como uma matriz em compósito (Ghosh et al., 2010). Em geral, o PLA tem uma boa barreira de propriedade e uma boa óptica física e mecânica, sendo considerado um método poderoso para a obtenção de características específicas de uso final (Murariu \& Dubois, 2016).

As propriedades do PLA, no entanto, são mutáveis a depender da cristalinidade, estrutura do polímero, peso molecular, formação de material e, principalmente, do processamento, o que possibilita uma extensa versatilidade física, variando de um plástico macio e elástico até um plástico rígido e de alta resistência (Ghosh et al., 2010; Madhavan Nampoothiri et al., 2010). 
Atualmente, o PLA representa um dos materiais mais populares no âmbito da manufatura aditiva (MA) - processo de fabricação por meio da adição de material em camadas planas e sucessivas (Volpato, 2017) associado, especificamente, ao processo de modelagem por fusão e deposição (FDM) (Santana et al., 2018), o qual envolve, através da extrusão de material, diferentes estratégias de preenchimento, onde, camada por camada, o filamento fino do material extrudado entra em contato com o material da superfície da peça, solidifica-se e adere à camada anterior (Sai Chennakesava \& Yeole, 2014; Volpato, 2017).

O comportamento mecânico das peças finais, produzidas em FDM, são influenciados pelos variados parâmetro de impressão, uma vez que sua mesoestrutura apresenta uma densidade de vazios pela existência de ligações filamento-filamento nas camadas e entre elas (Alves et al., 2021; Bellini \& Güçeri, 2003). Neste quesito, os ensaios de tração fornecem dados quantitativos que determinam coeficientes importantes na análise do comportamento destes materiais, como o limite da resistência à tração, limite de escoamento, módulo de elasticidade, módulo de resiliência, módulo de tenacidade, ductilidade, coeficiente de encruamento e coeficiente de resistência (Azevedo et al., 2016; Lopes, 2011).

De fato, a versatilidade imposta pelo material PLA potencializa-se quando associada a um processo de fabricação com diversas perspectivas, e a análise do comportamento mecânico dessas peças torna-se essencial para que a escala de produção atinja novos paradigmas de incorporação. Nesse contexto, o objetivo deste estudo foi caracterizar a performance do material PLA quando manufaturado pela tecnologia FDM.

\section{Metodologia}

Trata-se de uma pesquisa de caráter experimental, de abordagem quantitativa a qual se baseia em estudos numéricos por meio do uso de medições de grandeza (Pereira et al., 2018), realizada no Laboratório de Tecnologias Tridimensionais (LT3D) alocado no Núcleo de Tecnologias Estratégicas em Saúde (NUTES), localizado na Universidade Estadual da Paraíba (UEPB).

Para a elaboração e organização do procedimento experimental, este estudo foi construído de acordo com a norma ASTM D3039/3039-95A, utilizando 12 conjuntos de ensaios com três amostras de características iguais para cada um, resultando, assim, em um total de 36 corpos de prova ensaiados.

Apesar da ASTM D638 (Método padrão de teste para Propriedades de tração de plásticos) ser considerada a norma padrão para os ensaios de tração de materiais poliméricos (Ahn et al., 2002), a escolha da ASTM D3039/3039-95A como norma de referência para aplicação dos ensaios de tração desta pesquisa firmou-se pela peculiaridade da estrutura interna das peças fabricadas em MA por meio do processo de FDM, uma vez que se assemelham a uma estrutura de fibra reforçada e utilizam carga axial ao longo das fibras durante os ensaios, caracterizando-se como estrutura sanduíche.

Os corpos de prova que deram seguimento a essa pesquisa foram elaborados através do processo de fabricação FDM, utilizando o material PLA e respeitando a norma de referência, a qual definiu uma geometria retangular com dimensões de $25 \mathrm{~mm}$ de largura, $90 \mathrm{~mm}$ de comprimento e 3,04 mm de espessura, conforme a Figura 1.

Figura 1. Geometria e Dimensões dos corpos de prova.
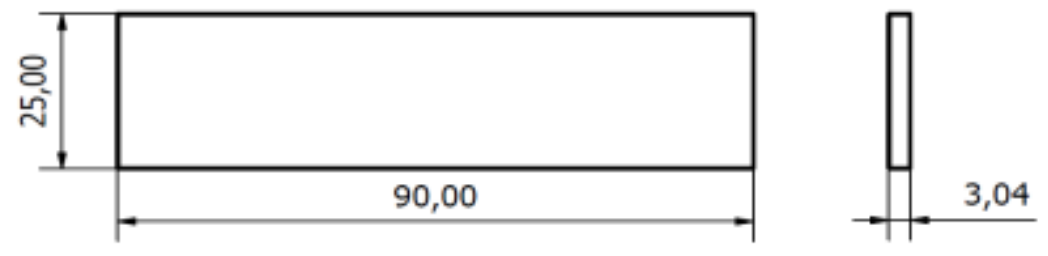

Fonte: ASTM D3039/3039-95', 1998. 
Os corpos de prova foram modelados através do software CAD Autodesk Inventor e, posteriormente, exportados em extensão .STL para o software CAM Makerware onde definiu-se os parâmetros e processos para a impressão 3D, tais como: Temperatura de extrusão; Posição do corpo de prova na bandeja de impressão; Loops (contorno do perímetro); Estratégia de preenchimento da camada (raster); Número de camadas e larguras de deposição; Taxa de deposição de preenchimento da camada; Velocidade de impressão; Ângulo de deposição do raster ( $\theta$ ); Temperatura da base da bandeja de impressão.

No que se refere a temperatura de extrusão, com o intuito de observar mudanças no comportamento mecânico das peças, os parâmetros utilizados foram $200^{\circ} \mathrm{C}$ e $215^{\circ} \mathrm{C}$, baseado no intervalo de temperatura estabelecido pelo fabricante do material PLA $\left(195^{\circ} \mathrm{C}\right.$ a $\left.220^{\circ} \mathrm{C}\right)$. Seguindo a mesma perspectiva, foi definido o desligamento para a bandeja de impressão e $120 \mathrm{~mm} / \mathrm{s}$ para velocidade de impressão, mantendo o padrão já estabelecido pela máquina.

Para o posicionamento dos corpos de prova foi considerado as posições dos eixos $\mathrm{X}$ e $\mathrm{Y}$, conforme indicado por Bagsik (2011). Vale ressaltar que para essa pesquisa não foi possível o posicionamento Z, devido a necessidade de produtos de aderência para fixação dos corpos de prova na bandeja de impressão. Visando manter a padronização, todos os corpos de prova foram posicionados no centro da bandeja de impressão.

Foi determinado o loop com perímetro de três para peças fabricadas no eixo $\mathrm{Y}$ e de dois para peças no eixo $\mathrm{X}$. As estratégias de preenchimento adotadas em cada camada foram do tipo estrutura retilínea com o ângulo de raster $(\theta)$ à $45^{\circ}$.

$\mathrm{O}$ número de camadas foi gerado automaticamente pelo software Makerware respeitando o sentido de adição de camadas em $\mathrm{Z}+$, assim, o processo variou de acordo com a posição do corpo de prova na bandeja. Diante disto, para o eixo $\mathrm{X}$ foi determinado 100 camadas sobrepostas e, para o eixo Y, 12 camadas. Por fim, três variáveis foram estabelecidas para a análise da taxa de deposição de preenchimento de camadas sendo, respectivamente, $20 \%, 50 \%$ e $100 \%$ de preenchimento interno estrutural.

Todos os parâmetros definidos para a pesquisa foram sintetizados na Tabela 1.

Tabela 1. Parâmetros utilizados na construção dos corpos de prova.

\begin{tabular}{lc}
\hline \multicolumn{1}{c}{ PARÂMETROS } & DESCRIÇÃO \\
\hline Temperatura de Extrusão & $200{ }^{\circ} \mathrm{C} ; 215^{\circ} \mathrm{C}$ \\
\hline Loops & 2 e 3 \\
\hline Estratégia de preenchimento (Raster) & Retilínea \\
\hline Número de camadas & Eixo Y: 12; Eixo X: 100 \\
\hline Taxa de deposição de preenchimento & $20 \% ; 50 \% ; 100 \%$ \\
\hline Velocidade de impressão & $120 \mathrm{~mm} / \mathrm{s}$ \\
\hline Ângulo do raster & $45^{\circ}$ \\
\hline Temperatura da bandeja impressão & $0{ }^{\circ} \mathrm{C}$ \\
\hline Largura da camada de deposição & $0,254 \mathrm{~mm}$ \\
\hline Diâmetro do Filamento PLA & $1,75 \mathrm{~mm}$ \\
\hline
\end{tabular}

Fonte: Autores. Dados da Pesquisa (2020).

Os ensaios de tração foram realizados na máquina EMIC DL 3000, utilizando uma célula de carga de $2.000 \mathrm{kgf}$ e uma taxa de deformação de $50 \mathrm{~mm} / \mathrm{min}$. As medições de deformações foram estabelecidas no software Tesc, sendo $50 \mathrm{~mm}$ de distância entre as garras de fixação dos corpos de prova, $25 \mathrm{~mm}$ de largura e 3,04 $\mathrm{mm}$ de espessura. Antes de todos os ensaios de tração foi mantida uma condição padrão com o laboratório a uma temperatura em torno de $20^{\circ} \mathrm{C}$. 
A descrição dos parâmetros do processo FDM para cada ensaio de tração, foi descrito na Tabela 2.

Tabela 2. Descrição dos parâmetros do processo FDM para os ensaios de tração.

\begin{tabular}{|c|c|c|c|c|c|c|c|}
\hline $\begin{array}{c}\text { Ensaios } \\
\text { (Conjuntos) }\end{array}$ & $\begin{array}{c}\text { Temperatura } \\
\text { Extrusão }\end{array}$ & Loop & $\begin{array}{l}\text { Posição } \\
\text { do Eixo }\end{array}$ & $\begin{array}{l}\text { Estratégia de } \\
\text { preenchimento }\end{array}$ & $\begin{array}{c}\text { Taxa de } \\
\text { deposição }\end{array}$ & $\begin{array}{c}\text { Espessura } \\
\text { camada }\end{array}$ & $\begin{array}{c}\text { Quantidade } \\
\text { de corpos de } \\
\text { prova }\end{array}$ \\
\hline 1 & $200^{\circ} \mathrm{C}$ & 3 & $\mathrm{Y}$ & Retilíneo & $20 \%$ & $0,254 \mathrm{~mm}$ & 3 \\
\hline 2 & $200^{\circ} \mathrm{C}$ & 3 & $\mathrm{Y}$ & Retilíneo & $50 \%$ & $0,254 \mathrm{~mm}$ & 3 \\
\hline 3 & $200^{\circ} \mathrm{C}$ & 3 & $\mathrm{Y}$ & Retilíneo & $100 \%$ & $0,254 \mathrm{~mm}$ & 3 \\
\hline 4 & $200^{\circ} \mathrm{C}$ & 2 & $\mathrm{X}$ & Retilíneo & $20 \%$ & $0,254 \mathrm{~mm}$ & 3 \\
\hline 5 & $200^{\circ} \mathrm{C}$ & 2 & $\mathrm{X}$ & Retilíneo & $50 \%$ & $0,254 \mathrm{~mm}$ & 3 \\
\hline 6 & $200^{\circ} \mathrm{C}$ & 2 & $\mathrm{X}$ & Retilíneo & $100 \%$ & $0,254 \mathrm{~mm}$ & 3 \\
\hline 7 & $215^{\circ} \mathrm{C}$ & 3 & $\mathrm{Y}$ & Retilíneo & $20 \%$ & $0,254 \mathrm{~mm}$ & 3 \\
\hline 8 & $215^{\circ} \mathrm{C}$ & 3 & $Y$ & Retilíneo & $50 \%$ & $0,254 \mathrm{~mm}$ & 3 \\
\hline 9 & $215^{\circ} \mathrm{C}$ & 3 & $\mathrm{Y}$ & Retilíneo & $100 \%$ & $0,254 \mathrm{~mm}$ & 3 \\
\hline 10 & $215^{\circ} \mathrm{C}$ & 2 & $\mathrm{X}$ & Retilíneo & $20 \%$ & $0,254 \mathrm{~mm}$ & 3 \\
\hline 11 & $215^{\circ} \mathrm{C}$ & 2 & $X$ & Retilíneo & $50 \%$ & $0,254 \mathrm{~mm}$ & 3 \\
\hline 12 & $215^{\circ} \mathrm{C}$ & 2 & $\mathrm{X}$ & Retilíneo & $100 \%$ & $0,254 \mathrm{~mm}$ & 3 \\
\hline \multicolumn{7}{|c|}{ TOTAL DE AMOSTRAS (CORPO DE PROVA) } & 36 \\
\hline
\end{tabular}

Fonte: Autores. Dados da Pesquisa (2020).

Visando adquirir um único valor significativo, para analisar os resultados dos ensaios de tração, foram calculadas as médias e os desvios padrões das três amostras de cada conjunto. Os valores definidos como significativos para este estudo foram: Tensão Máxima; Tensão de Ruptura e Módulo de Elasticidade.

\section{Resultados e Discussão}

Os corpos de prova manufaturados apresentaram comportamento anisotrópico, ou seja, diferentes propriedades mecânicas para cada eixo de construção, como já previstos na literatura (Camargo et al., 2019; Lovo \& Fortulan, 2016). Acredita-se que essa característica esteja relacionada à geometria dos vazios e a extensão da união entre filamentos individuais, estando diretamente ligada à magnitude de resistência desenvolvida pelas peças produzidas por MA (Santana, 2019).

Devido a necessidade de grandes quantidades de camadas de deposição para o eixo X em relação ao eixo Y, notou-se uma variação de tempo na construção dos corpos de prova entre as taxas de deposição 20\%, 50\% e 100\%, sendo, respectivamente, $16 \mathrm{~min}, 18 \mathrm{~min}$ e $20 \mathrm{~min}$ para o eixo Y e $54 \mathrm{~min}$, $56 \mathrm{~min}$, $58 \mathrm{~min}$ para o eixo X.

Nos testes de tensão-deformação, foi observado homogeneidade entre as três amostras de cada conjunto. As rupturas dos corpos de prova ocorreram em perfeito rompimento de perfil $\left(45^{\circ}\right)$ caracterizado por meio da deposição do ângulo raster $\left(\theta=45^{\circ}\right)$, como demonstrado na Figura 2. 
Figura 2. Rompimento dos corpos de prova após os ensaios de tração.

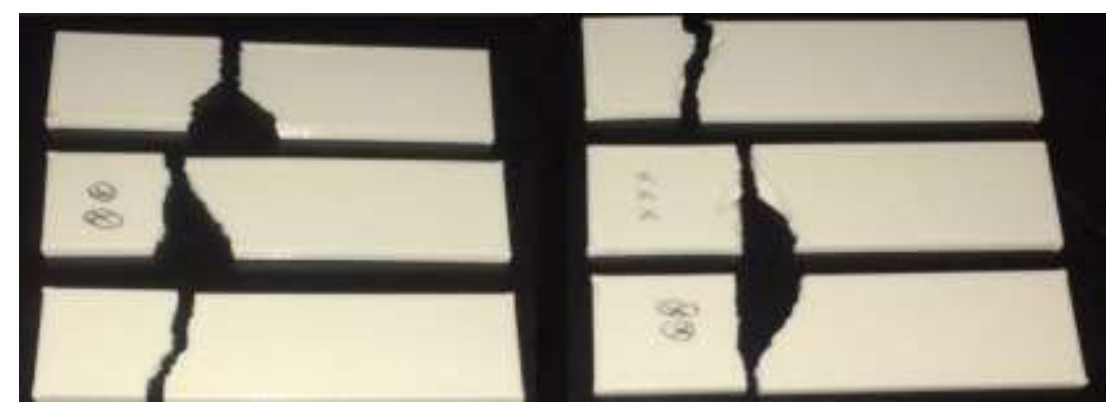

Fonte: Dados da Pesquisa (2020).

As curvas tensão-deformação nos corpos de provas, apresentaram-se praticamente sem área de escoamento (Gráfico 1 e Gráfico 2), o que define um comportamento tipicamente de materiais frágeis poliméricos. Em uma curva comum de tensãodeformação, a identificação do escoamento nem sempre é direta, ou seja, com uma perceptível separação entre o fim do comportamento elástico (relação linear) e o início do comportamento plástico (relação não linear) (Navarro, 2018).

Para Callister (2013) a fragilidade dos plásticos é influenciada pela redução de temperatura, no entanto, uma diferença de temperatura de $15^{\circ} \mathrm{C}$ não se mostrou significante para este estudo e, consequentemente, não apresentou influência no comportamento mecânico das peças. As curvas tensão-deformação na temperatura de extrusão a $200^{\circ} \mathrm{C}$ podem ser observadas no Gráfico 1. 
Research, Society and Development, v. 10, n. 8, e44210817348, 2021

(CC BY 4.0) | ISSN 2525-3409 | DOI: http://dx.doi.org/10.33448/rsd-v10i8.17348

Gráfico 1. Curvas de tensão-deformação dos corpos de prova com temperatura de extrusão a $200^{\circ} \mathrm{C}$
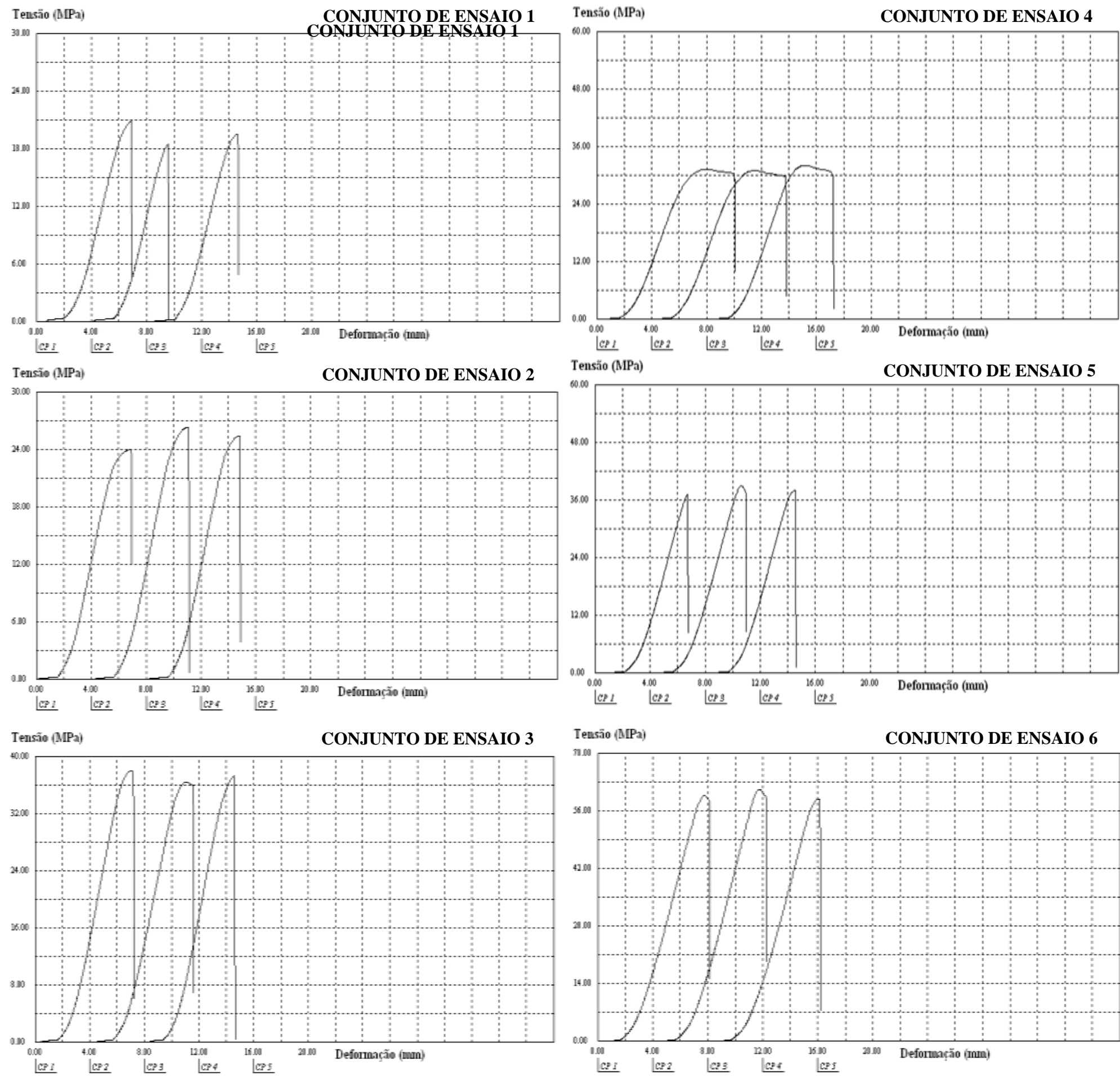

Fonte: Autores. Dados da Pesquisa (2020).

As curvas de tensão-deformação relacionadas a temperatura de extrusão de $215^{\circ} \mathrm{C}$ podem ser observadas no Gráfico 2 . 
Gráfico 2. Curvas de tensão-deformação dos corpos de prova com temperatura de extrusão $215^{\circ} \mathrm{C}$.

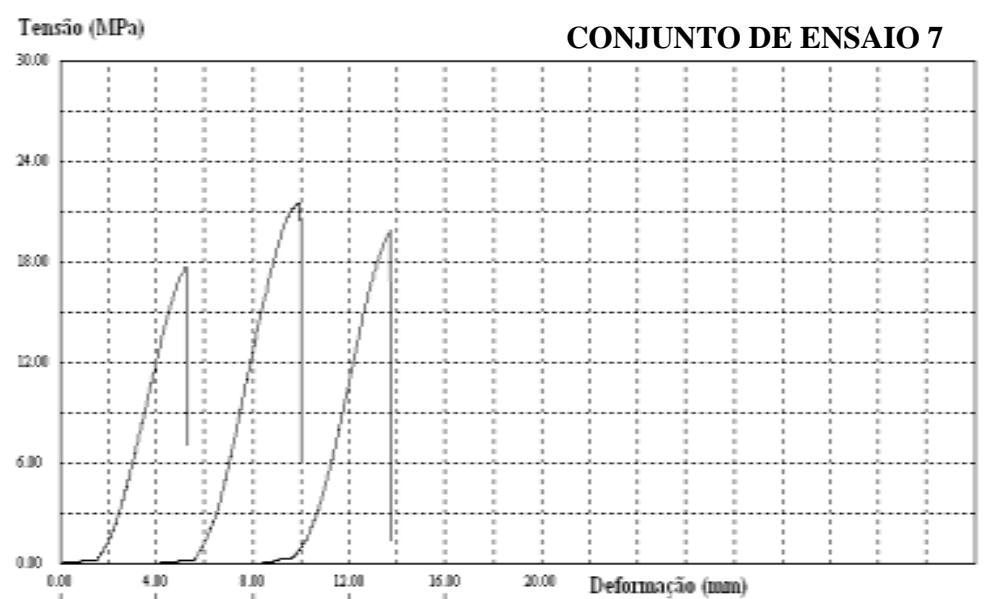

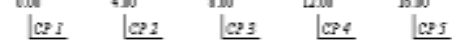

$$
\text { Tensăo (MPa) }
$$

CONJUNTO DE ENSAIO 8

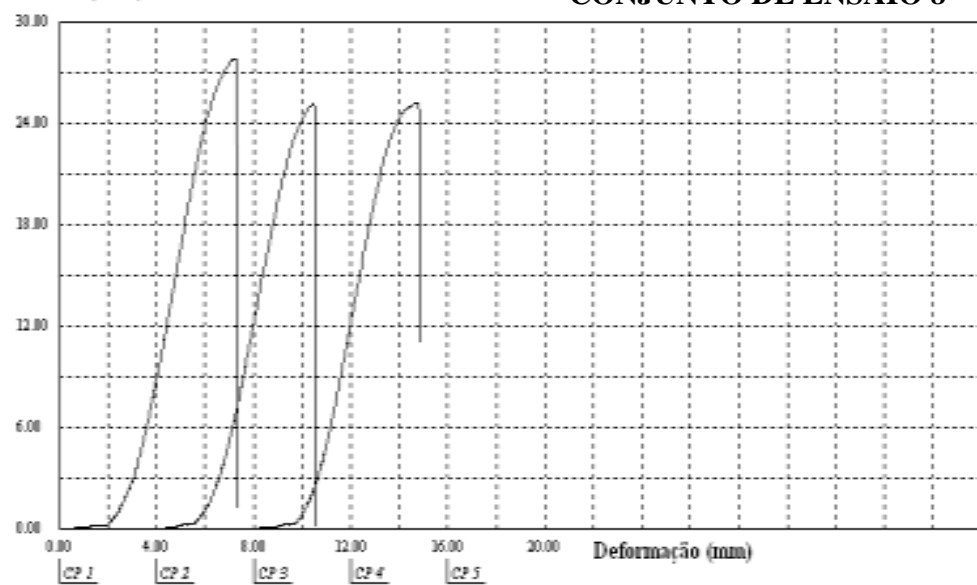

$$
\text { Teนsล̃o (MPa) }
$$

CONJUNTO DE ENSAIO 9
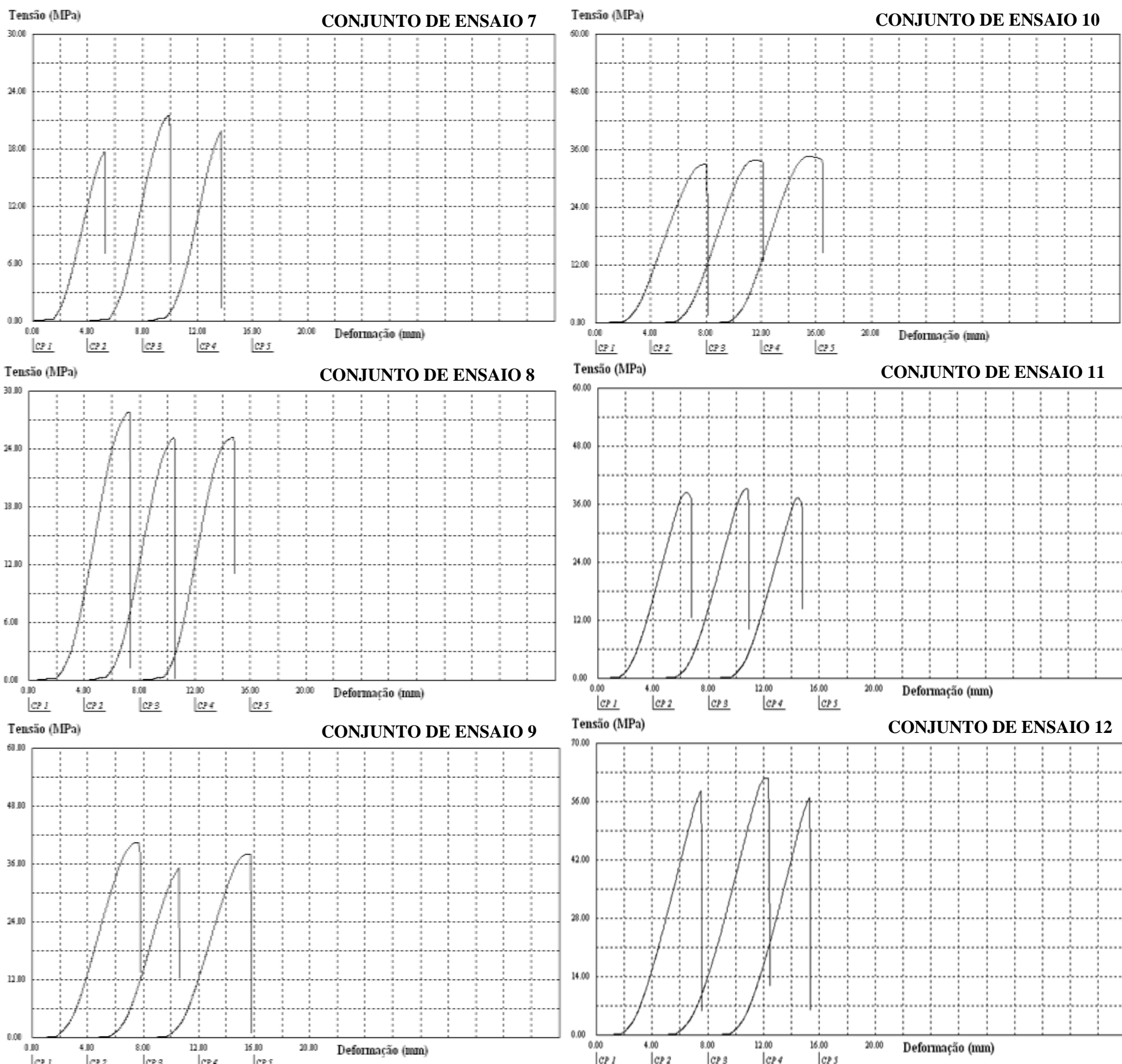

Tensăo (MPa)

CONJUNTO DE ENSAIO 11

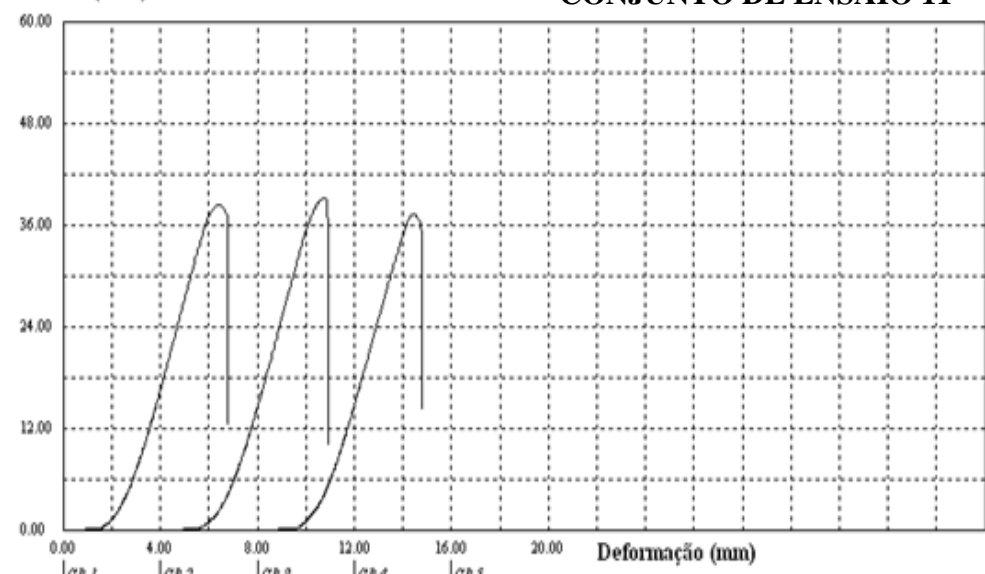

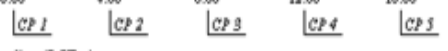

Tensăo $(\mathrm{MPa})$

CONJUNTO DE ENSAIO 12

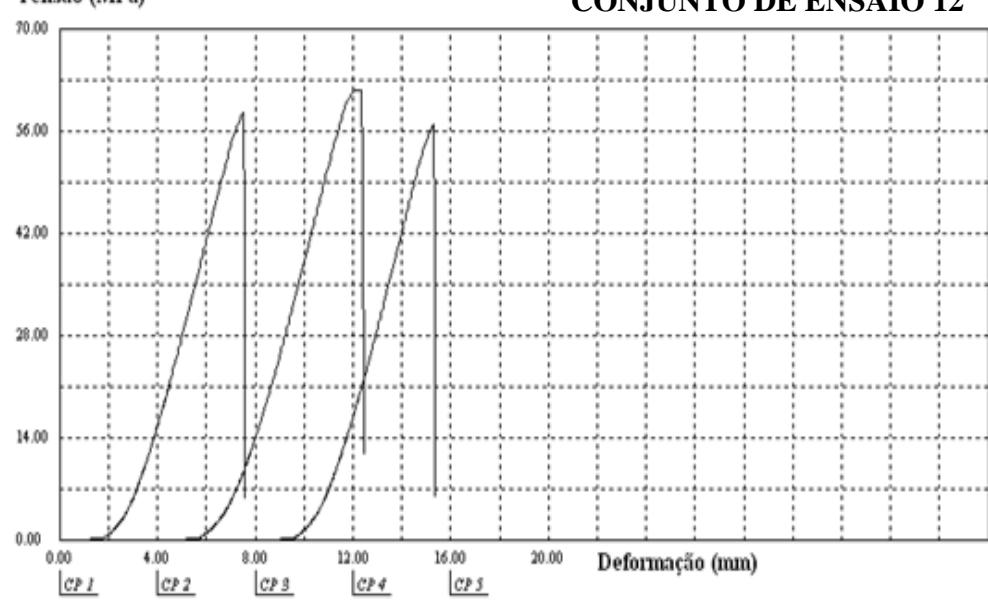

Fonte: Autores. Dados da Pesquisa (2020).

As taxas de deposição de 100\% para o eixo X (Conjuntos 6 e 12) apresentaram maiores índices para tensão de ruptura em contraposto as taxas de deposição de $20 \%$ do eixo Y (Conjuntos 1 e 7) que apresentaram os menores índices. Assim, percebe-se que quanto maior a área de preenchimento, maior será a tensão máxima e a tensão de ruptura, consequentemente maior serão os módulos de elasticidade e mais rígida será a peça.

No que se refere ao alongamento, as peças com $20 \%$ de taxa de deposição no eixo X (Conjuntos 4 e 10) apresentaram maiores índices, corroborando com Bagsik (2011) que observou maior resistência e alongamento antes da ruptura nas amostras 
de testes construídas na direção de eixo X. Além disso, notou-se que os mesmos (Conjuntos 4 e 10) apresentaram uma área de escoamento mais aparente, o que os difere dos demais conjuntos de ensaios.

Para tensão máxima o valor mais significativo nos testes mecânicos (465.4 ะ9.29) está relacionado com o eixo X, que obteve uma taxa de deposição de $100 \%$ e uma temperatura de extrusão de $200^{\circ} \mathrm{C}$ (Conjunto 6). As propriedades mecânicas, resistência à tração e resistência à flexão, aumentaram conforme o preenchimento, concordando com outros autores na literatura (Camargo et al., 2019; Heidari-Rarani et al., 2019; Torres et al., 2015).

As propriedades mecânicas dos corpos de prova foram sintetizadas na Tabela 3.

Tabela 3. Propriedades Mecânicas dos corpos de provas submetidos ao teste de tração.

\begin{tabular}{|c|c|c|c|c|c|c|c|}
\hline Conjunto & $\begin{array}{c}\text { Taxa de } \\
\text { Deposição }\end{array}$ & $\begin{array}{c}\text { Força Máxima } \\
\text { F[kgf] }\end{array}$ & $\begin{array}{c}\text { Tensão } \\
\text { Máxima } \\
\text { Máx[MPa] }\end{array}$ & $\begin{array}{c}\text { Tensão } \\
\text { Ruptura } \\
\text { Rup [MPa] }\end{array}$ & $\begin{array}{c}\text { Alongamento } \\
(\%)\end{array}$ & $\begin{array}{c}\text { Tensão } \\
\text { Escoamento } \\
\text { e[MPa] }\end{array}$ & $\begin{array}{c}\text { Módulo de } \\
\text { Elasticidade } \\
\text { E[MPa] }\end{array}$ \\
\hline \multicolumn{8}{|c|}{$200^{\circ} \mathrm{C}$ para eixo $\mathrm{Y}$} \\
\hline 1 & $20 \%$ & $152.6 \pm 9.15$ & $19.69 \pm 1.18$ & $19.63 \pm 1.22$ & $12.60 \pm 1.41$ & $17.47 \pm 0.69$ & $305.5 \pm 11.14$ \\
\hline 2 & $50 \%$ & $196.2 \pm 9.17$ & $25.32 \pm 1.18$ & $25.30 \pm 1.19$ & $13.83 \pm 0.31$ & $21.94 \pm 1.29$ & $361.7 \pm 7.30$ \\
\hline 3 & $100 \%$ & $289.3 \pm 6.09$ & $37.33 \pm 0.79$ & $37.21 \pm 0.96$ & $14.12 \pm 0.90$ & $33.94 \pm 0.60$ & $496.9 \pm 18.39$ \\
\hline \multicolumn{8}{|c|}{$200^{\circ} \mathrm{C}$ para eixo $X$} \\
\hline 4 & $20 \%$ & $245.0 \pm 4.18$ & $31.61 \pm 0.54$ & $30.15 \pm 0.31$ & $19.22 \pm 0.71$ & $26.74 \pm 1.43$ & $407.7 \pm 21.04$ \\
\hline 5 & $50 \%$ & $295.8 \pm 6.98$ & $38.17 \pm 0.90$ & $37.69 \pm 0.53$ & $13.41 \pm 0.44$ & $37.03 \pm 0.74$ & $538.4 \pm 3.59$ \\
\hline 6 & $100 \%$ & $465.4 \pm 9.29$ & $60.05 \pm 1.20$ & $59.17 \pm 0.40$ & $16.24 \pm 0.16$ & $58.31 \pm 1.61$ & $651.2 \pm 15.74$ \\
\hline \multicolumn{8}{|c|}{$215^{\circ} \mathrm{C}$ para eixo $\mathrm{Y}$} \\
\hline 7 & $20 \%$ & $153.0 \pm 14.71$ & $19.75 \pm 1.90$ & $19.72 \pm 1.92$ & $11.22 \pm 0.74$ & $17.57 \pm 1.12$ & $328.5 \pm 18.13$ \\
\hline 8 & $50 \%$ & $202.6 \pm 11.69$ & $26.14 \pm 1.51$ & $26.13 \pm 1.49$ & $13.67 \pm 0.75$ & $22.25 \pm 1.71$ & $385.5 \pm 8.95$ \\
\hline 9 & $100 \%$ & $294.4 \pm 20.55$ & $37.99 \pm 2.65$ & $37.92 \pm 2.76$ & $14.58 \pm 1.32$ & $33.32 \pm 2.30$ & $493.2 \pm 18.63$ \\
\hline \multicolumn{8}{|c|}{$215^{\circ} \mathrm{C}$ para eixo $X$} \\
\hline 10 & $20 \%$ & $263.0 \pm 6.34$ & $33.93 \pm 0.82$ & $33.57 \pm 0.50$ & $16.33 \pm 0.38$ & $29.20 \pm 1.56$ & $418.2 \pm 17.82$ \\
\hline 11 & $50 \%$ & $297.8 \pm 7.03$ & $38.42 \pm 0.91$ & $37.85 \pm 1.27$ & $13.50 \pm 0.16$ & $37.00 \pm 0.50$ & $531.7 \pm 13.71$ \\
\hline 12 & $100 \%$ & $458.5 \pm 19.19$ & $59.16 \pm 2.48$ & $58.92 \pm 2.70$ & $15.33 \pm 1.12$ & $57.74 \pm 2.01$ & $660.4 \pm 0.37$ \\
\hline
\end{tabular}

Fonte: Autores. Dados da Pesquisa (2020).

\section{Considerações Finais}

O material PLA, manufaturado pelo processo de FDM, demonstrou melhor performance mecânica quando produzido em eixo X com taxas de deposição de $100 \%$ para o preenchimento interno estrutural e temperatura de extrusão de $200^{\circ} \mathrm{C}$. Apesar de um aumento de tempo considerável para construção dos corpos de provas, os valores de tensão máxima, tensão de ruptura e módulo elástico foram mais significativos nas peças produzidas com orientação no eixo X.

Um aspecto importante observado através das curvas de tensão-deformação, advindas dos ensaios de tração, foram as modificações significativas das propriedades mecânicas do material pela influência de sensíveis alterações no processamento, caracterizando o PLA como um dos típicos materiais frágeis poliméricos.

Os resultados encontrados nesta pesquisa fortalecem a literatura sobre as características da performance do PLA e fornecem subsídios para que estudos futuros sejam realizados sobre o comportamento desse material no eixo $\mathrm{Z}$ a partir do desenvolvimento de novas estratégias de impressão que não apresentem vieses nos estudos. 


\section{Referências}

Ahn, S. H., Montero, M., Odell, D., Roundy, S., \& Wright, P. K. (2002). Anisotropic material properties of fused deposition modeling ABS. In Rapid Prototyping Journal (8, 4). https://doi.org/10.1108/13552540210441166

Alves, T. P., Jesus, A. V. S. De, Valverde, G., Ribeiro, R. R. M., Kívia, L., \& Rodrigues, D. O. (2021). Análise Da Influência Do Padrão De Preenchimento E Da Altura De Camada De Deposição Na Tensão De Ruptura De Corpos De Prova Fabricados Em Poli Ácido Lático (Pla) A Partir De Impressão 3 D.

ASTM D303995a (1998). Standard test method for tensile properties of polymer matrix composite materials.

Azevedo, C. M. P. de, Godinho, L. H., Saraiva, M. A. L., Alvarenga, S. das D., \& Ribeiro, M. V. A. de A. (2016). Ensaio Mecânico De Tensão-Deformação Sob Tração: Um Estudo Integrado Com A Disciplina De Cálculo. Perspectivas Online: Exatas \& Engenharia, 29-35.

Bagsik, A. (2011). Mechanical Properties Of Fused Deposition Modeling Parts Manufactured With Ultem. $1294-1298$.

Bellini, A., \& Güçeri, S. (2003). Mechanical characterization of parts fabricated using fused deposition modeling. Rapid Prototyping Journal, 9(4), 252-264. https://doi.org/10.1108/13552540310489631

Camargo, J. C., Machado, Á. R., Almeida, E. C., \& Silva, E. F. M. S. (2019). Mechanical properties of PLA-graphene filament for FDM 3D printing. International Journal of Advanced Manufacturing Technology, 103(5-8), 2423-2443. https://doi.org/10.1007/s00170-019-03532-5

Callister, Jr. (2013). Ciências e engenharia dos materias: uma introdução. (LTC (8 ed.))

Ghosh, S. B., Bandyopadhyay-Ghosh, S., \& Sain, M. (2010). Composites. Poly(Lactic Acid): Synthesis, Structures, Properties, Processing, and Applications, 293-310. https://doi.org/10.1002/9780470649848.ch18

Heidari-Rarani, M., Rafiee-Afarani, M., \& Zahedi, A. M. (2019). Mechanical characterization of FDM 3D printing of continuous carbon fiber reinforced PLA composites. Composites Part B: Engineering, 175(October 2018), 107147. https://doi.org/10.1016/j.compositesb.2019.107147

Lopes, J. T. de B. (2011). Propriedades Dos Materiais: Diagramas Tensão-Deformação. In Estrutura e Propriedades dos Materiais (pp. 126-161).

Lovo, J. F. P., \& Fortulan, C. A. (2016). Estudo de propriedades mecânicas e anisotropia em peças fabricadas por manufatura aditiva tipo FDM. I Simpósio Do Programa de Pós-Graduação Em Engenharia Mecânica Da EESC-USP (SiPGEM/EESC-USP), 1, 2-8.

Madhavan Nampoothiri, K., Nair, N. R., \& John, R. P. (2010). An overview of the recent developments in polylactide (PLA) research. Bioresource Technology, 101(22), 8493-8501. https://doi.org/10.1016/j.biortech.2010.05.092

Murariu, M., \& Dubois, P. (2016). PLA composites: From production to properties. Advanced Drug Delivery Reviews, 107, 17-46. https://doi.org/10.1016/j.addr.2016.04.003

Navarro, R. F. (2018). Comportamento plástico e os efeitos do Envelhecimento sobre a tensão de escoamento. Revista Eletrônica de Materiais e Processos, 3 , $170-176$.

Pereira, A., Shitsuka, D., Parreira, F., \& Shitsuka, R. (2018). Método Qualitativo, Quantitativo ou Quali-Quanti. In Metodologia da Pesquisa Científica. https://repositorio.ufsm.br/bitstream/handle/1/15824/Lic_Computacao_Metodologia-Pesquisa-Cientifica.pdf?sequence=1. Acesso em: 28 março 2020.

Sai Chennakesava, P., \& Yeole, S. (2014). Fused Deposition Modeling. July. https://doi.org/10.1201/9780203910795.ch8

Santana, L. (2019). Avaliação Das Capacidades Da Impressão 3D De Baixo Custo Na Fabricação De Snap-Fits: Uma Relação De Reconhecimento UsuárioSistema De Impressão. International Journal of Structural Integrity, 1(2), 161-172.

Santana, L., Alves, J. L., Sabino Netto, A. da C., \& Merlini, C. (2018). A comparative study between PETG and PLA for 3D printing through thermal, chemical and mechanical characterization. Revista Materia, 23(4). https://doi.org/10.1590/s1517-707620180004.0601

Torres, J., Cotelo, J., Karl, J., \& Gordon, A. P. (2015). Mechanical property optimization of FDM PLA in shear with multiple objectives. Jom, 67(5), 11831193. https://doi.org/10.1007/s11837-015-1367-y

Volpato, N. (2017). Manufatura Aditiva: Tecnologias e aplicações na impressão 3D. 